\title{
Non-Mammalian Models to Study Infections of the Central Nervous System and to Screen Potential Therapeutic Agents
}

\author{
Naveed Ahmed Khan* and Ruqaiyyah Siddiqui
}

School of Veterinary Medicine and Science, University of Nottingham, England, UK

\begin{abstract}
Infections of the central nervous system present the most significant challenge in our attempt to combat infectious diseases. Part of our inability to counter these diseases is attributed to an incomplete understanding of (i) how infectious agents cross the blood-brain barrier, a key step in the development of CNS infections, and (ii) the inability of drugs to traverse the blood-brain barrier to gain entry into the brain to kill parasites. Thus, there is an urgent need to expedite both drug discovery and drug delivery programmes. Here, we describe the use of in vitro, ex vivo, and in vivo models of the blood-brain barrier to examine the physical barriers, cellular mechanisms and molecular elements participating from both sides of the parasite-host interactions. In addition, these models provide useful screening tools to give rough indications of the potential of new antimicrobial agents with respect to efficacy, optimal routes of administration, and toxicity.
\end{abstract}

Keywords: Blood-brain barrier, in vivo models, in vitro models, locusts, human brain microvascular endothelial cells.

\section{INTRODUCTION}

The study of infectious diseases is a matter of great concern regarding human health. Protozoal, bacterial, fungal and viral opportunistic infections are becoming more prevalent with the overuse of antibiotics and the growing HIV pandemic. The failure of the blood-brain barrier structural integrity and function plays a fundamental role in the pathogenesis of many diseases of the central nervous system (CNS). For example, the free-living amoeba, Acanthamoeba are able to cause infections of the CNS that almost always lead to death. It is thought to be more deadly than other parasitic infections such as malaria, as death is unavoidable if a patient is untreated. Similarly, CNS infections such as cerebral tuberculosis due to Mycobacterium spp., cerebral malaria due to Plasmodium falciparum and African sleeping sickness due to Trypanosoma spp. have re-emerged as major causes of morbidity and mortality [1]. A complete understanding of the underlying pathogenic mechanisms of parasite invasion of the CNS and identification of novel approaches to screen and enhance antimicrobial transport across the blood-brain barrier should promote the efficiency of the treatment of diseases, and will identify novel targets for potential therapeutic interventions. This report describes recently developed non-mammalian host models to (i) elucidate determinants involved in the core processes of breaching the blood-brain barrier, and (ii) to screen potential antimicrobial compounds to combat diseases caused by neuropathogens. Although vertebrate models are seen as immediately more relevant, the proposed models can offer several advantages in terms of speed, cost, technical convenience, and ethical acceptance.

*Address correspondence to this author at the School of Veterinary Medicine and Science, University of Nottingham, England, UK; Tel: +44 (0) 115951 6409; Fax: +44 (0) 1159516440 ;

E-mail: Naveed.Khan@nottingham.ac.uk

\section{Model of the Blood-Brain Barrier, In Vitro}

Representative, reproducible, and high-throughput models of human cells and tissues are necessary for a significant evaluation of pathogen-host interactions .The most informative infection models: animals, organ explants, and human trials are not suitable for identification of molecular determinants involved in the pathogenic mechanisms and basic screening of candidate drugs. To this end, in vitro models of the blood-brain barrier are undoubtedly vital tools to identify the cellular and molecular elements that should provide targets for interventions of the transmigration of pathogens as well as small molecules into the CNS [2]. Specialized brain microvascular endothelial cells that line the intraluminal portion of the brain capillaries form the blood-brain barrier. Tight junction proteins that prevent leakage of blood-borne substances into the brain parenchyma characterize these endothelial cells. This makes the blood-brain barrier highly selective compared with the peripheral endothelium [3]. The tight junction proteins are primarily responsible for the barrier function, preventing entry of blood contents into the CNS [3]. The tight junctions consist of integral membrane proteins (i.e., occludin, claudin, junction adhesion molecule, endothelial cell-selective adhesion molecules), and cytoplasmic proteins, zonula occludens-1 (ZO-1), ZO-2, ZO-3. Occludin is a phosphoprotein and its cytoplasmic domain is directly associated with zonula proteins. Claudins bind to claudins on the adjacent endothelial cells to form the tight junctions. The carboxy terminal of claudins binds to the cytoplasmic $\mathrm{ZO}$ proteins. Claudin-5 and claudin-3 are localized in the tight junctions of the brain endothelial cells. Junctional adhesion molecules and the endothelial cell-selective adhesion molecules also are localized in the tight junctions of the blood-brain barrier. The accessory proteins of the $\mathrm{ZO}$ provide structural support and bind to integral membrane proteins of the tight junctions, further linking membrane proteins to the actin cytoskeleton [4-6]. A key feature to 
measure the integrity of the blood-brain barrier is that it exhibits high transendothelial electrical resistance, TEER (approximately 2,000 ohm/ $\mathrm{cm}^{2}$ ) [7], while endothelial cells from the human placenta exhibit less than $50 \mathrm{ohm} / \mathrm{cm}^{2}$ [8]. The high selectivity ensures that even small molecules such as dyes and antibiotics are prevented from entry into the $\mathrm{CNS}$, while non-brain endothelium is more permissive.

The failure of the blood-brain barrier structural integrity and function plays an essential role in the pathogenesis of many diseases of the CNS. Therefore, during ischemia, inflammation, trauma, neoplasia, hypertension, and epilepsy, altered blood-brain barrier permeability is frequently observed [9]. The availability of primary human brain microvascular endothelial cells (HBMEC), in vitro has been of incredible value in the study of parasite-host interactions [10,11]. The HBMEC are isolated, characterized, and purified from the cerebral cortex [11] and tested for endothelial markers such as factor VIII, carbonic anhydrase IV, Ulex Europaeus Agglutinin I, gamma-glutamyl transpeptidase, and uptake of acetylated low-density lipoprotein. In addition, these cells demonstrated the expression of tight junction proteins and the formation of a polar monolayer. The HBMEC are routinely grown on rat-tail collagen-coated dishes in complete medium [RPMI 1640 containing $10 \%$ heat-inactivated fetal bovine serum, 10\% Nu-Serum, $2 \mathrm{mM}$ glutamine, $1 \mathrm{mM}$ pyruvate, penicillin (100 U/ml), streptomycin $(100 \mu \mathrm{g} / \mathrm{ml})$, non-essential amino acids and vitamins] [10,11]. The model of the blood-brain barrier, in vitro is developed by cultivating HBMEC in the upper chamber of collagen-coated Transwells polycarbonate tissue culture inserts, (Fig. (1)), and grown for at least 2 days. Following this incubation, the media are replaced with fresh culture media containing $500 \mathrm{nM}$ hydrocortisone and cultured for an additional 3-6 days. Under these conditions, HBMEC monolayers exhibit tight junction formation and polarization and develop TEER of more than $200 \mathrm{ohm} / \mathrm{cm}^{2}$

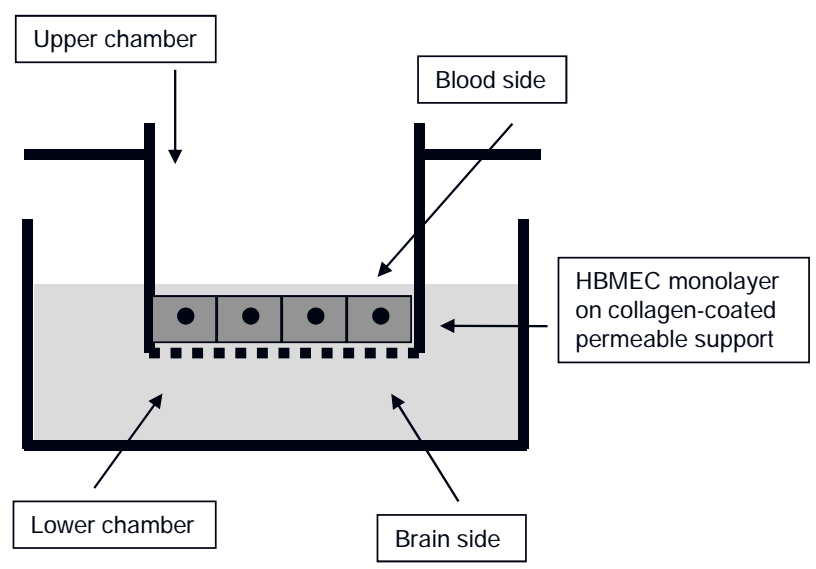

Fig. (1). Model of the blood-brain barrier, in vitro. The polarized in vitro model is formed by growing brain microvascular endothelial cells to confluency as a monolayer on transwell inserts. Labelled pathogens/molecules can be added in the upper chamber (blood-side). After various intervals of time, the contents of the lower chamber (brain-side) can be removed to determine traversal of the blood-brain barrier. using a tissue-resistant measurement chamber and a voltohmmeter. Using this in vitro model, our studies have shown that Acanthamoeba is able to transmigrate the bloodbrain barrier by modulating the integrity of the HBMEC [12]. This was demonstrated by findings that Acanthamoeba interactions with HBMEC increased monolayer permeability and abolished TEER, indicating that amoebae induced blood-brain barrier perturbations [12].

It is interesting that Acanthamoeba conditioned medium degraded occludin and $\mathrm{ZO}$ proteins suggesting that amoebae target the paracellular route to disrupt the blood-brain barrier. In addition, the findings revealed that Acanthamoeba conditioned medium induces activation of Rho-associated intracellular signaling cascades in HBMEC [12]. Rhoassociated pathways could disturb the function of tight junctions, thus leading to increased blood-brain barrier permeability. For example, recent studies have shown that RhoA regulates phosphorylation of myosin light-chain phosphorylation causing structural changes and redistribution of ZO-1 and occludin leading to increased blood-brain barrier permeability [13]. In addition to the examination of toxic effects on HBMEC, traversal of the monolayer can also be studied by inoculating pathogens/ antimicrobials to the upper chamber and by monitoring their traversal across the monolayer over various intervals of time. Subsequently, samples are taken from the bottom chamber to determine percent crossing of the original inoculum.

As well as studying disease pathogenesis, this is a useful model to test novel drug therapies, in vitro. An ex vivo model of the human blood-brain barrier has also been developed. This model makes use of astrocytes, pericytes, neurones and the HBMEC in Transwells [1,14].

\section{Non-Mammalian Model of the Blood-Brain Barrier, Ex Vivo}

Ex vivo models are advantageous as experiments can be performed using living tissue derived from a whole organism and work can be conducted in a controlled environment. Although, their usefulness is limited, as they require a constant supply of live tissues, ex vivo models are more relevant than in vitro models, as whole tissues are used and infections can be studied in a relatively real situation. They are particularly relevant to study infections of tissues such as the CNS. Given the ethical concerns associated with the use of vertebrates, we have recently reported the use of invertebrates to develop a novel ex vivo model of the bloodbrain barrier [15]. To achieve this, locusts were ligated in the neck region using a loop of fine thread placed between the head and thorax, and tied to allow the head capsule to be removed from the body by cutting just behind the ligature using fine scissors. Although ligation prevented leakage of material from the cut end of the gut, the back of the head capsule was sealed using wax to ensure that no leakage of haemolymph from the head capsule could occur if the neck membrane had been damaged during ligation. The isolated head capsule contained an intact and undamaged brain, and the blood-brain barrier remained intact within the capsule; damage to the CNS was limited to the cutting of the ventral nerve cord, the cut ends of which were sealed by the thread and the wax outside the isolated head capsule, (Fig. (2)). 
A)

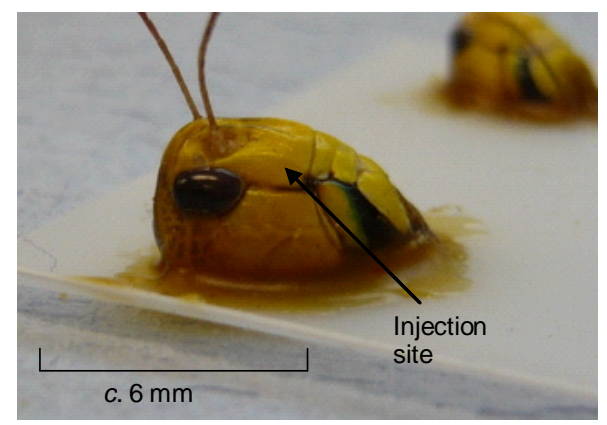

B)

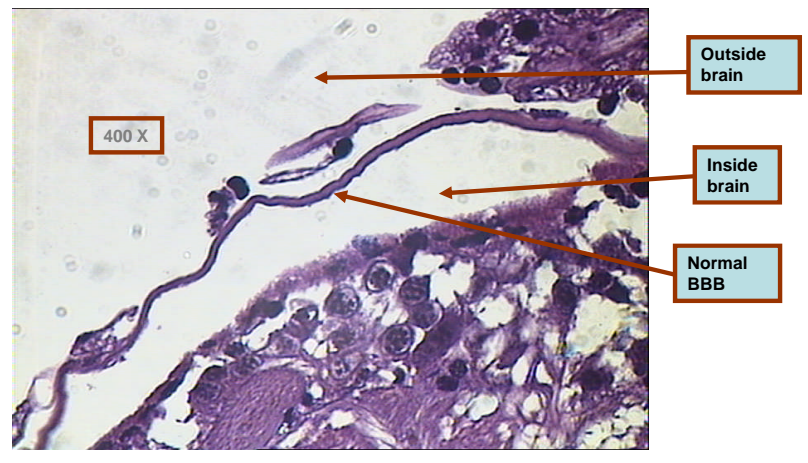

Fig. (2). Head capsules are removed by ligating locusts in the neck region using a loop of fine thread. The back of the head capsule is sealed using wax (A). In this way, the isolated head capsule contained an intact and undamaged brain, and the bloodbrain barrier remains intact within the capsule, as demonstraded by hematoxylin and eosin staining of the sectioned brains $(\mathbf{B})$.

In this way, the ability of pathogen/molecule crossing of the blood-brain barrier could be studied. Using this model, we demonstrated that virulence determinants that are involved in mammalian infection are important for Escherichia coli K1 traversal of the locust blood-brain barrier. Such a model offers several advantages over existing mammalian models in relation to its comparative ease of use, cost-effectiveness and ethical acceptability.

\section{Non-Mammalian Model of the Blood-Brain Barrier, In Vivo}

A whole-organism approach to the study of disease is crucial in gaining a complete understanding of the interrelationships between infectious agents and their hosts. At present, most research involving human pathogens involves mammalian hosts including rodent, simian, and feline hosts. For example, animal models that have been used for investigating Acanthamoeba encephalitis include mice, rats and non-human primates such as monkeys [1620]. In addition to the considerable ethical concerns over mammalian suffering, studies using these host systems are time-consuming and animal maintenance can be costly. Such models may not be routinely available in many laboratories. Thus the replacement of insects for mammals as model hosts for the study of human opportunistic pathogens has a number of advantages. There are remarkable parallels between the innate immune responses of mammals and insects [21], particularly as insects such as locusts possess a highly selective blood-brain barrier exhibiting functional properties comparable with that of vertebrates [22], (Fig. (3)), indicating that insects make a useful model to study the microbial crossing of the blood-brain barrier, in vivo.
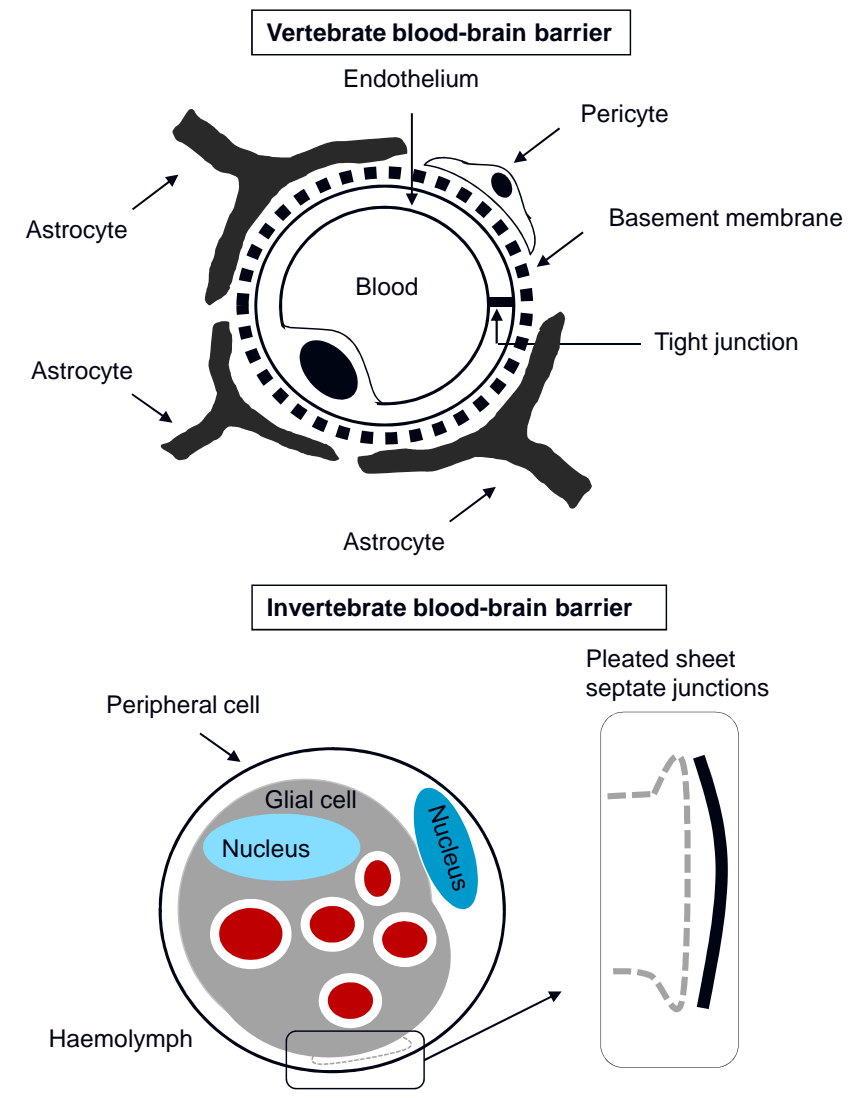

Fig. (3). Anatomical similarities of the blood-brain barrier of vertebrates and invertebrates.

To this end, our recent studies have shown the use of locust as a novel in vivo model of the blood-brain barrier [6]. Injection of Acanthamoeba into the haemocoel of the locust abdomen by inserting the needle into the intersegmental membrane between two abdominal terga produced locust death with almost $100 \%$ mortality. To determine parasite association with the CNS, locust brains are isolated by removing the left side of head by a saggital cut and the brain dissected out using fine forceps. Care is taken to ensure that both cerebral ganglia are present and that they are free of fat body tissue and air sacs, and surgical instruments are surface-sterilised with methylated spirit between dissections, (Fig. (4)).

Histological examination of the CNS showed that Acanthamoeba invaded the locust CNS, and this is associated with disruption of the perineurium cell/glial cell complex, which constitutes the locust blood-brain barrier, (Fig. (5)). Thus, it is tempting to speculate that Acanthamoeba-mediated locust death is, in part, associated with the parasite's ability to invade the locust CNS.

Post-injection, viable amoebae were recovered from the locust haemolymph, as well as other tissues such as fat body and muscle tissues, suggesting that Acanthamoeba produced parasitaemia; survived the onslaught of the innate immune 


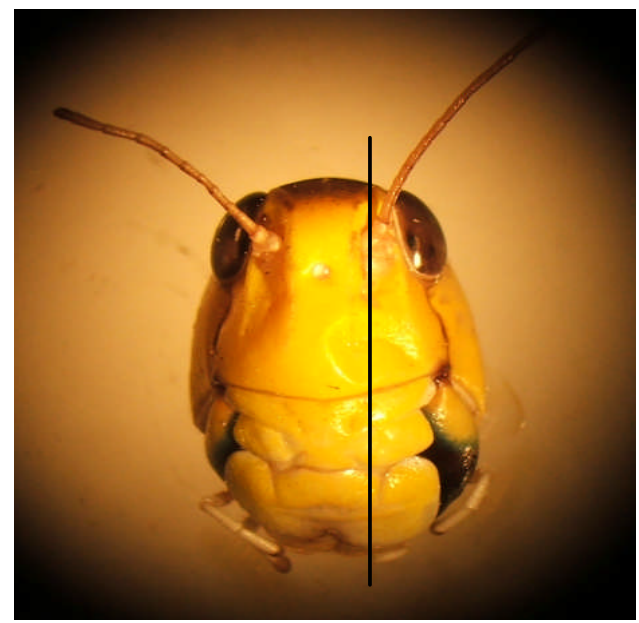

Locusts are killed and head capsules are removed from the body. Left side of head is removed by a saggital cut.

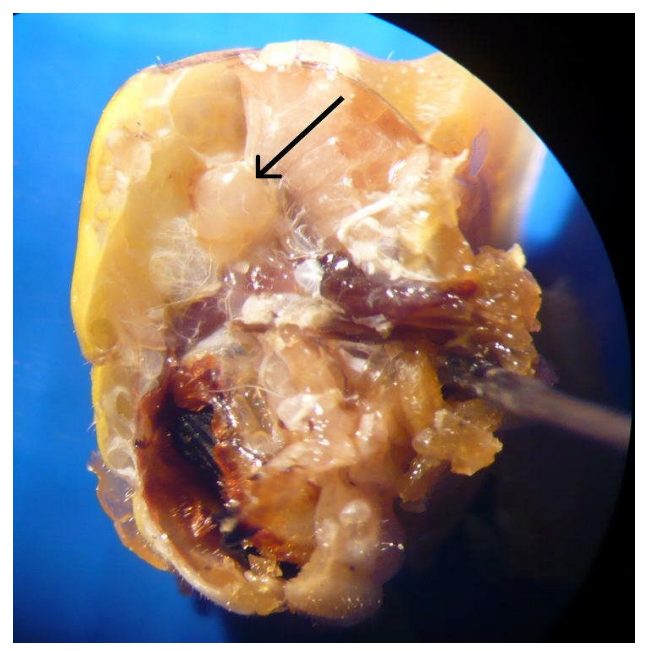

Brains are dissected out using forceps

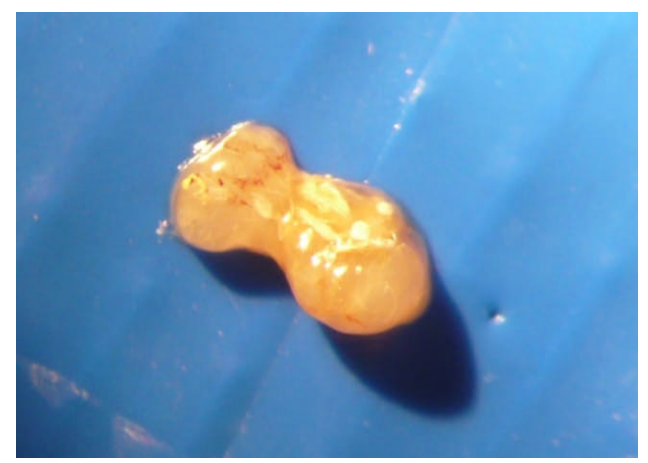

Intact brains are isolated

Fig. (4). Locusts as an in vivo model to study infections of the central nervous system. Brains can be isolated to study microbial invasion of the central nervous system.

defences of locusts; disseminated to other tissues and invaded the CNS, a finding that is consistent with the human form of Acanthamoeba encephalitis. It is noteworthy that the molecular components of the blood-brain barrier in insects such as Drosophila are homologous to those of vertebrates [23], thus it is possible that there will be similarities between the mechanisms by which microbes invade the CNS of mammals and that of locusts, and research is underway to investigate these issues.

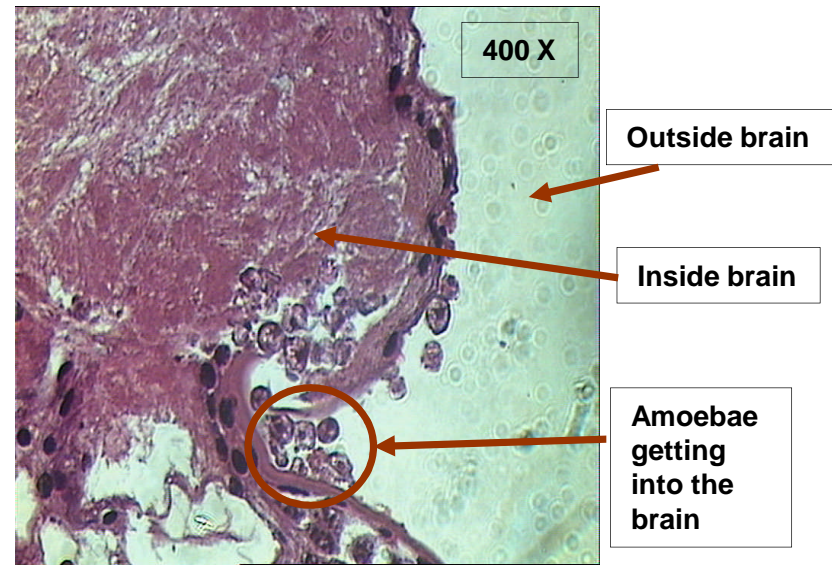

Fig. (5). Locusts were injected with $10^{6}$ amoebae and brains dissected out 7 days post-infection. Subsequently, brains were sectioned and stained with hematoxylin and eosin. Note that amoebae-injected locusts showed the infiltration of parasites into the brain tissue as shown by arrows.

On the whole, the in vivo model developed in this study is rapid, reproducible, relatively easy to perform, and can be used to investigate microbial invasion of the CNS. Even though vertebrate model systems may be seen as more relevant, the aforementioned models are valuable in rapidly screening large numbers of genes/determinants of interest for microbial pathogenesis that can be tested consequently in mammalian systems, thus reducing the numbers of mammals required overall [15].

In addition to studying microbial pathogenesis, such models also have the scope in exploring the efficacy of novel drugs (testing large chemical libraries) in microbial diseases, allowing inexpensive, rapid, and even high-throughput experimentation. Given that pharmacokinetic profiles of potential antimicrobial agents would undoubtedly differ between humans and locusts, i.e., the latter having an open circulatory system that would eliminate compounds faster, the results should be interpreted with caution and compared with results obtained from the clinical studies. As long as, such differences are recognized, locusts can be exploited for predicting pharmacokinetics in vertebrates. Despite these differences, these basic screening models give rough indications of the potential of new antimicrobial agent with respect to efficacy, optimal routes of administration, and toxicity.

\section{ACKNOWLEDGEMENTS}

The authors are grateful to Mary Lightfoot, Graham Goldsworthy, Khadijo Osman, Parisa Mortazavi, Behzad Mokri-Moayyed (Birkbeck, University of London), for their sincere help and kind support in developing locust model, and to Kwang Sik Kim and Monique Stins (Johns Hopkins 
University) for their help in developing the blood-brain barrier model.

\section{ABBREVIATIONS}

CNS $=$ Central nervous system

$\mathrm{ZO}=$ Zonula occludens

TEER $=$ Transendothelial electrical resistance

HBMEC = Human brain microvascular endothelial cells

\section{REFERENCES}

[1] Khan, N. A. Acanthamoeba Biology and Pathogenesis. Caister Academic Press: UK, 2009.

[2] Kong, H. H.; Seo, S. A.; Shin, C. O.; Im, K. I. The effect of active immunization with Acanthamoeba culbertsoni in mice born to immune mother. Korean J. Parasitol., 1993, 31, 157-163.

[3] Miller, D. W. Immunobiology of the blood-brain barrier. $J$. Neurovirol., 1999, 5, 570-578.

[4] Huber, J. D.; Egleton, R. D.; Davis, T. P. Molecular physiology and pathophysiology of tight junctions in the blood-brain barrier. Trend. Neurosci., 2001, 24, 719-725.

[5] Kim, J. H.; Kim, J. H.; Park, J. A.; Lee, S.; Kim, W. J.; Yu, Y. S.; Kim, K. Blood-neural barrier: intercellular communication at gliovascular interface. J. Biochem. Mol. Biol., 2006, 39, 339-345.

[6] Tuomanen, E. Entry of pathogens into the central nervous system. FEMS Microbiol. Rev., 1996, 18, 289-299.

[7] Pardridge, W. M. Blood-brain barrier: biology and methodology. $J$. Neurovirol., 1999, 5, 556-569.

[8] Jinga, V. V.; Gafencu, A.; Antohe, F.; Constantinescu, E.; Heltianu, C.; Raicu, M.; Manolescu, I.; Hunziker, W.; Simionescu, M. Establishment of a pure vascular endothelial cell line from human placenta. Placenta, 2000, 21, 325-336.

[9] Gerald, A.; Grant, N.; Abbott, J.; Janigro, D. Understanding the Physiology of the Blood-Brain Barrier: In vitro Models. News Physiol. Sci., 1998, 13, 287-293.

[10] Alsam, S.; Kim, K. S.; Stins, M.; Rivas, A. O.; Sissons, J.; Khan, N. A. Acanthamoeba interactions with human brain microvascular endothelial cells. Microb. Pathog., 2003, 35, 235-241.
[11] Stins, M. F.; Gilles, F.; Kim, K. S. Selective expression of adhesion molecules on human brain microvascular endothelial cells. $J$. Neuroimmunol., 1997, 76, 81-90.

[12] Khan, N.A.; Siddiqui, R. Acanthamoeba affects the integrity of human brain microvascular endothelial cells and degrades the tight junction proteins. Int. J. Parasitol., 2009, 13, 1611-1616.

[13] Shen, L.; Black, E, D.; Witkowski, E. D.; Lencer, W. I.; Guerriero, V.; Schneeberger, E. E.; Turner, J. R. Myosin light chain phosphorylation regulates barrier function by remodeling tight junction structure. J. Cell. Sci., 2006, 119, 2095-2106.

[14] Khan, N. A. Acanthamoeba: Biology and Pathogenesis, Science Publishers: Enfield, UK, 2009.

[15] Mokri-Moayyed, B.; Goldsworthy, G.; Khan, N. A. Development of a novel ex vivo insect model for studying virulence determinants of Escherichia coli K1. J. Med. Microbiol., 2008, 57, 106-110.

[16] Cabral, G. A.; Marciano-Cabral, F. Cannabinoid-mediated exacerbation of brain infection by opportunistic amebae. $J$. Neuroimmunol., 2004, 147, 127-130.

[17] Cerva, L. Intranasal, intrapulmonary and intracardial inoculation of experimental animals with Hartmanella castellanii. Folia Parasitol., 1967, 14, 207-215.

[18] Culbertson, C. G.; Smith, J. W.; Cohen, H. K.; Minner, J. R. Experimental infection of mice and monkeys by Acanthamoeba. Am. J. Pathol.,1959, 35, 185-197.

[19] El-Dein, S. Z.; Khalifa, A. M.; Sadaka, H. A.; Hegazy, I. H.; Ibrahim, H. S. Electroencephalographic changes in rats received antigens of different parasites. J. Egypt. Soc. Parasitol., 1998, 28 , 797-805.

[20] Hadaś, E.; Mazur, T. Biochemical markers of pathogenicity and virulence of Acanthamoeba sp. strains. Parasitol. Res., 1993, 79, 696-698.

[21] Scully, L. R.; Bidochka, M. J. Developing insect models for the study of current and emerging human pathogens. FEMS Microbiol. Lett., 2006, 263, 1-9.

[22] Carlson, S. D.; Juang, J. L.; Hilgers, S. L.; Garment, M. B. Blood barriers of the insect. Ann. Rev. Entomol., 2000, 45, 151-174.

[23] Willott, E.; Balda, M. S.; Fanning, A. S.; Jameson, B.; Van Itallie, C.; Anderson, J. M. The tight junction protein ZO-1 is homologous to the Drosophila discs-large tumor suppressor protein of septate junctions. Proc. Natl. Acad. Sci. USA, 1993, 90, 7834-7838.

(C) Khan and Siddiqui; Licensee Bentham Open.

This is an open access article licensed under the terms of the Creative Commons Attribution Non-Commercial License (http://creativecommons.org/licenses/by-nc/3.0/) which permits unrestricted, non-commercial use, distribution and reproduction in any medium, provided the work is properly cited. 